\title{
La construcción del "mundo de ficción" y de la trama narrativa en situaciones de juego simbólico en el hogar
}

\section{The Construction of the Fictional World and the Narrative Structure in Symbolic Play Situations in Home Settings}

\author{
Alejandra Stein ${ }^{1}$ \\ Maia Julieta Migdalek ${ }^{2}$ \\ Universidad de Buenos Aires - CONICET, Argentina
}

\begin{abstract}
Resumen. Con la participación de niños de sectores medios de Argentina, se presenta un estudio longitudinal acerca de la construcción del mundo ficcional y de la trama narrativa en situaciones de juego. Investigaciones previas evidenciaron las relaciones entre el juego y las habilidades narrativas. Son escasos los estudios que analizaron los recursos que contribuyen a elaborar el mundo ficcional del juego, así como las características de su trama narrativa. Se analizaron 60 situaciones de juego simbólico en las que participaron 12 niños y sus madres cuando los primeros tenían 2.6 y 3.6 años. Los participantes emplean diversos recursos para la construcción del mundo ficcional. Se observaron variaciones longitudinales respecto del uso de enunciados metalúdicos, el tipo de mundo ficcional y la complejidad de la trama narrativa del juego.
\end{abstract}

Palabras clave. Juego, lenguaje temprano, habilidades narrativas, estudio longitudinal.

Abstract. The paper presents a longitudinal analysis of the construction of the fictional world and the narrative discourse in play situations registered in the homes of middle-income children from Argentina. Previous research has shown the relations between play and narrative abilities. There are few studies focused on the characteristics of the interaction and the language that contribute to the narrative represented in the play. Sixty play situations were analyzed and registered in the homes of 12 children when they were 2.6 and 3.6 years old respectively. The participants used several resources for the construction of the fictional world of the play. Longitudinal variations were observed regarding the use of metaplay utterances, the types of fictional worlds constructed and the complexity of the narratives.

Keywords. Play, early language, narrative abilities, longitudinal study.

\footnotetext{
${ }^{1}$ Alejandra Stein. Universidad de Buenos Aires - Consejo Nacional de Investigaciones Científicas y Técnicas (CONICET), Argentina. Dirección postal: Tte. J. Perón 2158, CABA, CP.1040. E-mail: alejandrastein@yahoo.com.ar

${ }^{2}$ Maia Julieta Migdalek. Universidad de Buenos Aires - Consejo Nacional de Investigaciones Científicas y Técnicas (CONICET), Argentina. E-mail: maiamig@hotmail.com
}

\section{@ $\odot \Theta \Theta$}




\section{Introducción}

El presente trabajo se enmarca en una línea de investigación que aborda las relaciones entre el juego simbólico y el desarrollo lingüístico. El juego simbólico se define como el tipo de juego en el que el niño emplea un objeto, o el lenguaje, para servir como significante para representar otra entidad; el significado. Aquello que es representado puede ser cualquier aspecto de las experiencias vividas o imaginadas de los niños como roles sociales, situaciones, actividades u objetos (Garvey, 1985; Gönçü \& Gaskins, 2011; Sarlé \& Rosas, 2005). Al jugar con otros, los niños y sus compañeros emplean diversos recursos para crear el mundo ficcional y desplegar la trama lúdica, es decir, se valen de diversos medios para comunicarle a sus compañeros las identidades, los escenarios, las acciones y los objetos involucrados en la representación (Garvey, 1985). Tales elementos constitutivos del juego simbólico son característicos, también, de las narraciones (Pellegrini, 1985a).

El objetivo del presente trabajo consiste en analizar el mundo ficcional creado en situaciones de juego simbólico, los recursos empleados por los niños y sus madres para construirlo y las características de la trama narrativa representada durante el período comprendido entre los 2.6 y 3.6 años.

Diversos estudios han atendido a los cambios longitudinales en el juego simbólico. Mayormente, concuerdan en que el juego simbólico surge alrededor de los 18 meses y se desarrolla hasta los 7 años de edad (Elkonin, 1980; Garvey, 1985; Rakoczy, 2008; Westby, 1988). Durante el segundo año de vida, los niños comienzan a participar en situaciones de juego que involucran la creación de una esfera o realidad alternativa del "como si". Inicialmente, esta esfera es creada a partir de la simulación de acciones aisladas que conllevan alguna transformación simbólica -por ejemplo, una niña usa una cuchara como si fuera un peine- (Westby, 1988). El argumento de estas formas tempranas de juego se reduce a una secuencia de acciones reiterativas sin una secuencia narrativa que las ligue (Elkonin, 1980). Los papeles asumidos por los niños más pequeños se vinculan, principalmente, con roles familiares y actividades domésticas (Garvey \&
Berndt, 1977). En efecto, estudios recientes señalan el carácter social del juego simbólico, especialmente del juego de simulación temprano, en tanto se adquiere a través del aprendizaje imitativo cultural (Gönçü \& Gaskins, 2011; Rakoczy, 2007). Las tempranas representaciones de la vida cotidiana son andamiadas, iniciadas y, principalmente, llevadas a cabo por los padres de los niños (Rakoczy, 2007, 2008).

Alrededor del tercer año, los niños expanden las transformaciones simbólicas y las incluyen en juegos que dependen de un marco narrativo. La posibilidad de combinar acciones en el juego, creando una secuencia narrativa, se relaciona con la mayor comprensión que tienen los niños acerca de las interrelaciones entre las personas y los objetos (Garvey, 1985; Westby, 1988). En esta etapa, los niños también desarrollan habilidades narrativas -son capaces de usar el lenguaje para referirse a eventos desplazados en el tiempo y el espacio- (Westby, 1988). El desarrollo de estas habilidades les permite a los niños crear mundos ficcionales que imitan situaciones de la vida cotidiana o que involucran elementos fantásticos (Engel, 2005).

Alrededor de los tres años y medio, los niños comienzan a usar un lenguaje metacognitivo -yo pienso, me olvidé...- que se encuentra asociado a la habilidad para planificar. Ello, a su vez, les permite representar secuencias de acciones más elaboradas y organizadas (Westby, 1988). Paulatinamente, a medida que los niños van creciendo, asumen una mayor diversidad de roles, incluyendo actividades fantásticas (Garvey, 1985; Schwartzman, 1978). Asimismo, la situación representada en el juego va estando menos determinada por las propiedades de los materiales de los objetos presentes y va entrando, cada vez más, bajo el control de planes o ideas (Garvey, 1985). En el presente estudio se atenderá a las variaciones longitudinales en el tipo de mundo ficcional -cercano a la vida cotidiana versus fantástico- creado en el juego durante el período considerado.

La estrecha relación entre el juego y el lenguaje se evidencia tempranamente, incluso antes de la emergencia del juego simbólico. Los juegos de dar y tomar y de aparecer y desaparecer, observados 
durante el período de los 3 a 24 meses, constituyen actividades privilegiadas para el desarrollo de los actos de habla de petición y de referencia (Bruner, 1986). El juego aporta un formato en el cual los niños pueden comenzar a individualizar referentes, primero con formas idiosincrásicas, para luego pasar al uso de formas lingüísticas cada vez más cercanas a la forma convencional. En estas situaciones, los niños también comienzan a usar el lenguaje para solicitar objetos o ayuda para realizar una acción. Estos aprendizajes son facilitados por las características estables de la situación que provee el juego, en tanto formato, y al rol de la madre, quien provee un fuerte andamiaje inicial, que paulatinamente va retirando hasta lograr la autonomía del niño (Bruner, 1986).

El juego simbólico, en particular, también ha sido objeto de un conjunto de estudios que analizaron sus relaciones con el desarrollo de formas más complejas de lenguaje descontextualizado (Pellegrini, 1982, 1984, 1985a; Rosemberg, Arrúe \& Migdalek, 2015; Stein, Migdalek \& Sarlé, 2012), discurso argumentativo (Migdalek, Santibáñez \& Rosemberg, 2014) y narrativo (Pellegrini, 1985a). Dentro de esta línea, una serie de investigaciones atendieron a las relaciones entre el juego simbólico y el desarrollo de habilidades narrativas por parte de niños en edad preescolar (Fein, Ardila-Rein \& Goth, 2000; Galda, 1984; Nicolopoulou, Schnabel Cortina, Ilgaz \& Brockmeyer, 2015; Pellegrini, 1985a; Sachs, Goldman \& Chaille, 1984).

Estos trabajos pusieron de manifiesto la existencia de semejanzas entre el juego simbólico y la narrativa, tanto en su estructura como en el estilo de lenguaje empleado en ellos. Por un lado, tanto el juego simbólico como la narración han sido caracterizados como "dos expresiones distintivas del pensamiento simbólico" (Kavanaugh \& Engel, 1998, p. 81) dado que ambos implican la (re)creación de un contexto (Trionfi \& Reese, 2009). Durante el juego simbólico, para la creación de dicho contexto, los niños realizan transformaciones de objetos y transformaciones ideacionales -transformaciones más abstractas e independientes de los objetos presentes-. Para evitar la ambigüedad en el juego, estas transformaciones se explicitan verbalmente, lo cual implica un uso más complejo del lenguaje. Los niños deben recurrir a formas en las que priman las referencias endofóricas -los elementos que se nombran se definen y caracterizan dentro del texto y no por referencia al contexto de situación-. Asimismo, requieren la definición de los objetos y de los personajes por medio de frases nominales complejas que incluyen modificadores directos e indirectos. Para definir estas transformaciones con sus compañeros de juego de manera tal que el significado de la transformación no sea ambiguo, los niños deben emplear un estilo de lenguaje explícito, descontextualizado y con una sintaxis relativamente compleja (Feldman, 2005; Pellegrini, 1985b, 1986). Por otro lado, el juego y la narración presentan semejanzas en cuanto a su estructura, pues ambos involucran personajes ficcionales que operan en una realidad creada y contienen elementos tales como motivaciones y complicaciones. En efecto, en el juego simbólico los niños representan eventos fantásticos o de la vida cotidiana y, para ello, deben analizar y reconstruir la estructura temporal y causal de estos eventos, la cual es similar a la de las narraciones (Ilgaz \& Aksu-Koc, 2005; Nicolopoulou, McDowell \& Brockmeyer, 2006; Pellegrini, 1985a). En el presente trabajo se analizan las características que asume la trama narrativa en las situaciones de juego simbólico bajo estudio, así como sus variaciones longitudinales en el período comprendido entre los 2.6 y los 3.6 años.

Dadas las semejanzas entre juego y narración, una serie de estudios (Nicolopoulou et al., 2006; Nicolopoulou et al., 2015; Pellegrini, 1984; Pellegrini \& Galda, 1982) mostraron el impacto en el desarrollo de habilidades narrativas -comprensión, recuerdo y producción de narraciones- de los niños a partir de su participación en intervenciones que involucran situaciones de juego simbólico. En los trabajos de Nicolopoulou y colaboradores $(2006,2015)$-que a su vez están basados en experiencias de Paley (1990)-, un grupo de niños de 3 y 4 años participaron a lo largo de un año escolar de actividades que involucraban la producción de narraciones y su posterior representación en situaciones de juego. Los resultados pusieron de manifiesto un incremento significativo de 
las habilidades de comprensión de historias por parte de los niños del grupo experimental.

Por su parte, Pellegrini y Galda (1982) evaluaron la reconstrucción de un cuento por parte de niños preescolares y de segundo grado en tres condiciones -a partir de una situación de juego simbólico, de una conversación guiada por un adulto o de un dibujo-. Los resultados evidenciaron que para los niños de preescolar, la situación de juego simbólico fue el facilitador más eficaz para las diversas medidas de comprensión consideradas en el estudio. Los autores señalan que la representación de la situación narrada en el juego parece tener un efecto directo sobre la capacidad de volver a narrar una historia.

En la misma línea, Ilgaz y Aksu-Koc (2005) evaluaron la producción de un episodio narrativo por parte de niños de 3, 4 y 5 años en dos condiciones: 1) juego simbólico -creación de una historia mediante el empleo de una serie de juguetes- y 2) relato directo al experimentador -luego de pintar un libro con imágenes de animales, se solicitaba a los niños que le contaran una historia al experimentador; si los niños mostraban alguna dificultad para contar la historia, el experimentador les sugería que los personajes fueran los animales del libro para colorear-. Los resultados mostraron que los niños pudieron producir narrativas con componentes temporal-causal integrados a los 4 años en la condición de juego, mientras que, en la segunda condición, se registraron narrativas con tales características a los 5 años de edad. Estos resultados muestran el impacto que tienen las acciones situadas en actividades concretas para el desarrollo narrativo. Los autores concluyen que la competencia emergente de los niños para construir narrativas con estructura episódica se puede observar mejor en contextos de juego donde la acción y los objetos andamian tanto la organización conceptual como su expresión verbal.

Son menos numerosos los estudios que analizaron en detalle las características de la interacción y del lenguaje empleado en las situaciones lúdicas que pueden contribuir a la elaboración de la trama narrativa del juego (Garvey \& Kramer, 1989; Sawyer, 2002). En esta línea, el estudio de las situaciones de juego simbólico en las que participaron niños de 5 años (Sawyer, 2002) permitió identificar dos elementos que pueden contribuir a la elaboración narrativa: el uso de estrategias discursivas dialógicas -negociación del juego desde la voz de los personajes- y metalúdicas -habla por fuera de los personajes representados en el juego-. En estas situaciones, el conocimiento común de los participantes relativo a la situación que se está representando, es decir, el guión compartido (Nelson, 1996), también favorece la elaboración narrativa, en tanto provee una estructura lógico secuencial que los niños recrean en el juego (Ortega, 1994; Sawyer, 2002).

Mientras que los niños más pequeños se valen mayormente de la dramatización de acciones y el cambio de registro para el establecimiento del mundo ficcional (Gönçü, 1987; Rubin, Fein \& Vandenberg, 1983), los niños de 3 y 4 años de edad recurren a enunciados metalúdicos con expresiones tales como "Dale que ...". Se observó que el uso de este tipo de afirmaciones explícitas acerca del juego (Garvey, 1985), también llamadas emisiones metacomunicativas explícitas, se incrementa con la edad durante el período preescolar y luego disminuye alrededor de los 6 años (Andresen, 2005; Garvey \& Kramer, 1989). De acuerdo con Andresen (2005), estos hallazgos se explican a partir del proceso general de desarrollo ontogenético postulado por Vigotsky (1978), según el cual las emisiones metacomunicativas explícitas de los niños se internalizan y se vuelven implícitas. Se ha señalado, asimismo, la importancia de este tipo de enunciados para la vida social. Las transformaciones del tipo "dale que este bloque es una manzana" se establecen gracias a una intencionalidad colectiva, son altamente convencionales y crean un contexto normativo que rige las acciones futuras, rasgos todos de la vida institucional (Rakoczy, 2007, 2008).

En Argentina, Migdalek, Rosemberg y Arrúe (2013) analizaron el proceso de construcción del marco ficcional en el juego y el al uso de recursos lingüísticos en este proceso en situaciones lúdicas en las que participaron niños de nivel socioeconómico medio y bajo a los 4 años de edad. Los resultados mostraron tres tipos de construcción del marco ficcional en las 
situaciones de juego simbólico que se elaboran a partir de diferentes recursos discursivos: 1) situaciones en la que los niños se desplazan en dos planos -el mundo ficcional del juego y el mundo real- diferenciados explícitamente (Juego tipo I); 2) situaciones en las que, por medio de muñecos o personajes, la realidad ficcional se construye a través de acciones y lenguaje contextualizado en "el aquí y el ahora" (Juego tipo II); 3) situaciones en las que los niños se sitúan en un mundo ficcional sin establecer distinciones verbales, ni señalizar vínculos entre las actividades y los objetos del juego y los de la realidad (Juego tipo III).

Asimismo, en un trabajo posterior (2014) analizaron la relación entre el uso de ciertos recursos lingüísticos característicos de un tipo de discurso explícito y los distintos tipos de juego. Los resultados del análisis mostraron diferencias en el empleo por parte de los niños de perífrasis de obligación, cláusulas relativas, cláusulas sustantivas para establecer el marco ficcional, cláusulas sustantivas para identificar individuos, cláusulas adverbiales causales y cláusulas adverbiales finales, en función del tipo de juego. En particular se observaron diferencias significativas entre el juego de tipo I y los otros tipos de juego. Este tipo de juego, que se caracteriza por el hecho de que los niños se manejan simultáneamente en dos planos -el mundo ficcional del juego y el mundo real-, da lugar a que los niños empleen en mayor medida ciertos recursos discursivos para instaurar el juego, crear el contexto dramático y realizar las transformaciones de objetos necesarias para pasar del plano de la realidad al plano ficcional. Otro de los recursos empleados en la construcción del mundo ficcional identificado en las investigaciones antecedentes es el modelado. En este sentido, se observó que los niños despliegan una mayor cantidad de acciones dramáticas (pretend acts) luego del modelado de las acciones lúdicas por parte de un adulto (Leslie, 1987; Nielsen \& Christie, 2008). En este estudio se analizan los recursos específicos que los niños y sus interlocutores emplean para construir el mundo ficcional del juego. Adicionalmente, dada la relevancia de los enunciados metalúdicos que distinguen explícitamente el mundo ficcional del mundo real, se analizan las variaciones longitudinales en el empleo de este recurso.

En síntesis, los estudios previos atendieron al desarrollo del juego simbólico durante la infancia; estas investigaciones mostraron que los niños progresivamente pueden crear mundos ficcionales más complejos, con mayor cantidad de acciones integradas y distanciados de la vida cotidiana. Dadas las semejanzas entre los componentes del mundo ficcional del juego y la estructura de las narraciones, diversos trabajos analizaron las relaciones entre juego y desarrollo de habilidades narrativas.

Estos trabajos, o bien señalaron las semejanzas entre juego y narrativa, o bien analizaron las características específicas del lenguaje empleado en las situaciones de juego. Sin embargo, no atendieron en detalle a los recursos mediante los cuales se construye la trama del juego ni a su emergencia en el desarrollo. Tampoco atendieron en detalle a los componentes de la estructura narrativa del juego ni a sus variaciones longitudinales en el período considerado. Finalmente, son escasos los trabajos realizados en esta línea que involucraron a población de habla hispana (Auza, 2013). Por todo ello, en el presente trabajo se busca responder los siguientes interrogantes relativos al desarrollo del juego simbólico y a sus relaciones con el desarrollo del lenguaje, en particular, el discurso narrativo: ¿Qué tipos de mundo ficcional involucran los juegos en los que participan los niños a los 2.6 y a los 3.6 años? ¿Cuáles son los recursos mediante los cuales los niños y sus interlocutores construyen el mundo ficcional y la trama del juego? En relación con estos recursos, ¿se observa el uso de enunciados metalúdicos a esta edad? ¿Existen variaciones en su uso durante el período considerado? ¿Qué características asume la trama narrativa del mundo ficcional del juego en esta etapa?

\section{Método}

\section{Participantes}

Doce niños de sectores medios de la Ciudad de Buenos Aires, Argentina, junto con su madre, cuando estos tenían 2.6 y 3.6 años. Los progenitores de los niños cuentan con estudios universitarios completos. 
En dos de las situaciones registradas está presente el hermano mayor del niño. En uno de los casos, registrado a los 2.6, la hermana mayor promueve el desarrollo del juego de tomar el té mediante la dramatización de acciones y el cambio de registro. En el otro caso, registrado a los 3.6, el hermano mayor asume el rol de un personaje.

\section{Corpus}

El corpus está conformado por 60 situaciones de juego simbólico (34 situaciones cuando los niños tenían 2.6 y 26 situaciones a los 3.6 años) registradas en el contexto del hogar por medio de audio, video, o ambos, en las que participaron 12 niños y sus madres.

\section{Procedimientos de obtención y transcripción de la información empirica}

Se realizó una visita al hogar de cada niño cuando éste tenía 2.6 y 3.6 años de edad. En cada visita se indujeron situaciones de juego de las que participaron conjuntamente el niño y un miembro del hogar a cargo de su cuidado (que podía ser el padre, la madre, hermanos u otra persona a cargo del niño habitualmente en el hogar). En todos los casos considerados en este estudio, se trató de la madre del niño; ocasionalmente, también participaron hermanos. Las situaciones fueron inducidas mediante la introducción de una serie de juguetes apropiados para la edad de los participantes (autos, bloques grandes y pequeños, muñecos del tipo playmobil, set de vajilla para tomar el té, mamadera, teléfono celular).

Todas las situaciones fueron registradas con la presencia del observador. Con el objeto de intentar resguardar la validez ecológica de los datos no se pautó al interlocutor del niño. Tampoco se proporcionaron indicaciones relativas a la extensión de la situación ni a cómo interactuar con los niños.

Se tuvo acceso a las familias a través de contactos con personas conocidas. Con cada familia, se estableció un acuerdo de anonimato y confidencialidad. Los procedimientos metodológicos empleados cumplen con las normas éticas establecidas para la investigación científica en Ciencias Humanas y fueron evaluados a partir de informes presentados a las comisiones asesoras del Consejo Nacional de Investigaciones Científicas y Técnicas (CONICET).

Las situaciones fueron registradas por medio de audio y transcritas para su análisis. Los registros fueron complementados con las notas de campo elaboradas durante las observaciones.

\section{Procedimientos de análisis}

En el estudio se emplearon procedimientos de análisis cualitativo y cuantitativo. En ambos casos, la unidad de análisis está determinada por las situaciones de juego registradas, las cuales se distinguen entre sí en función del tema y los juguetes involucrados.

Se llevó a cabo un análisis cualitativo mediante el empleo del método comparativo constante (Glaser \& Strauss, 1967; Strauss \& Corbin, 1991) de los recursos empleados por los niños y sus interlocutores en la construcción del mundo ficcional del juego en los dos momentos considerados (2.6 y 3.6). En el marco de este análisis, se recurrió de modo heurístico a conceptos desarrollados en estudios previos (Garvey, 1987; Gönçü, 1987; Leslie, 1987; Nielsen \& Christie, 2008; Rubin, Fein \& Vandenberg, 1983; Sawyer, 2002). En la tabla 1 se incluyen las categorías empleadas en el análisis cualitativo.

Por su parte, con el fin de ahondar en el conocimiento del mundo ficcional construido en el juego a lo largo del tiempo, el análisis cuantitativo tuvo por objetivo identificar variaciones longitudinales. Se tomó en consideración el tipo de mundo ficcional creado en la situación lúdica, el uso de enunciados metalúdicos para la construcción del mundo ficcional, por parte de los niños y sus madres, y la estructura narrativa del juego.

El tipo de mundo ficcional creado en el juego. Se retomaron las categorías de Engel (2005), quien distingue dos tipos de mundos ficcionales creados en las situaciones de juego: un mundo ficcional que simula situaciones de la vida cotidiana (what is) y un mundo ficcional que comprende elementos fantásticos (what if). Se aplicó la prueba de Wilcoxon para muestras dependientes 
Tabla 1

Categorias empleadas en el análisis

\begin{tabular}{|c|c|c|}
\hline Categoría & Definición & Ejemplo \\
\hline Acción dramática & $\begin{array}{l}\text { Consiste en la representación de acción dentro } \\
\text { del mundo ficcional. }\end{array}$ & $\begin{array}{l}\text { Simula darle de tomar la leche en la } \\
\text { mamadera a la mamá) }\end{array}$ \\
\hline Diálogo ficcional & $\begin{array}{l}\text { Enunciados emitidos en el plano ficcional } \\
\text { desde la identidad del personaje. Aquí se } \\
\text { incluyen también los casos de cambio de } \\
\text { registro. }\end{array}$ & $\begin{array}{l}\text { Señora, ime prepara un té con leche por } \\
\text { favor? }\end{array}$ \\
\hline Explicitación de la acción & $\begin{array}{l}\text { Verbalizaciones que explicitan o especifican la } \\
\text { acción representada o aspectos de la misma. }\end{array}$ & Le pongo azúcar al té. \\
\hline Explicitación de guión & $\begin{array}{l}\text { Mención del guión propuesto para representar } \\
\text { en el juego. }\end{array}$ & Vamos a jugar al restaurant. \\
\hline Directivas & $\begin{array}{l}\text { Actos de habla destinados a dirigir la acción } \\
\text { del interlocutor. }\end{array}$ & Mové al muñeco. \\
\hline Referencia a experiencia personal & $\begin{array}{l}\text { Referencia a situaciones habituales o } \\
\text { experiencias pasadas vinculadas a la situación } \\
\text { lúdica. }\end{array}$ & $\begin{array}{l}\text { El papá (muñeco) va a hacer compras. Como } \\
\text { ayer, que fuiste con papi al supermercado. }\end{array}$ \\
\hline Enunciados metalúdicos & $\begin{array}{l}\text { Afirmaciones explícitas acerca del juego, } \\
\text { emitidas desde el plano no ficcional. }\end{array}$ & ¿Dale que yo era el papá y vos la mamá? \\
\hline
\end{tabular}

con el fin de estudiar las diferencias en el tipo de mundo ficcional representado en el juego según la edad de los niños.

El uso de enunciados metalúdicos. Los enunciados metalúdicos constituyen afirmaciones explícitas acerca del juego. Se identifican cuando los participantes hablan por fuera de los personajes representados -¿Dale que esta es la casa de los dragones?-. Según Sawyer (2002), el uso de enunciados metalúdicos contribuye a la elaboración de la trama narrativa. Se comparó la presencia de enunciados metalúdicos en las situaciones de juego registradas a los 2.6 y 3.6 años y se aplicó la prueba de Wilcoxon para muestras dependientes con el fin de estudiar las diferencias en la presencia de enunciados metalúdios según la edad de los niños.

La estructura narrativa. Para el análisis de la estructura narrativa del juego, se consideraron las categorías referidas a los elementos macroestructurales de las narraciones (Petersen, Gillam \& Gillam, 2008; Peterson \& McCabe, 1983; Stein \& Glenn, 1978, 1982). Se identificó la presencia de los siguientes componentes en las narrativas representadas en las situaciones lúdicas: identificación de personajes, caracterización de personajes, escenario, complicación, relaciones causales y de finalidad, relaciones temporales, solución o cierre. Se comparó la presencia de los elementos narrativos mencionados en las situaciones de juego registradas a los 2.6 y 3.6 años y se aplicó la prueba de Wilcoxon para muestras dependientes con el fin de estudiar las diferencias en la estructura narrativa según la edad de los niños.

Se utilizó como índice de acuerdo para la definición de las unidades de análisis (situaciones de juego) y para la codificación el coeficiente Kappa de Cohen. Los autores codificaron de manera independiente el $25 \%$ de los casos. Se registraron buenos índices de confiabilidad de observadores independientes en la identificación de situaciones de juego (acuerdo = $100 \%$ ), tipo de juego (acuerdo $=93.75 \%$; kappa $=$ .904), tipo de mundo ficcional (acuerdo = 93,75\%; 
kappa $=.846$ ), enunciados metalúdicos (acuerdo $=$ 93.75\%; kappa $=.871)$, ausencia de componentes estructurales narrativos (acuerdo $=87.5 \%$; $\mathrm{kappa}=$ .714), identificación de personajes (acuerdo $=87.5 \%$; kappa $=0.75$ ), caracterización de personajes (acuerdo $=93.75 \% ; \mathrm{kappa}=.871)$, escenario (acuerdo $=93.75 \%$; kappa $=.846)$, problema (acuerdo $=93.75 \%$; kappa $=, 846)$, relaciones causales y de finalidad (acuerdo $=$ $93.75 \%$; kappa $=.862$ ), secuencia temporal (acuerdo $=87.5 \%$; kappa $=.673$ ), solución o cierre (acuerdo $=93.75 \%$; kappa $=.765)$. Para el resto del corpus, se discutieron conjuntamente los casos problemáticos.

\section{Resultados}

Tanto a los 2.6 como a los 3.6 años, los niños involucrados en el presente estudio participaron junto con sus madres en situaciones de juego de "como si" en las que simularon tomar el té, preparar comida y hablar por teléfono con una persona conocida ausente o con un personaje de ficción, entre otros juegos. Algunos niños se involucraron también en juegos con muñecos u otros tipos de juegos simbólicos, tales como la dramatización breve de un cuento infantil, que conlleva la adopción, por parte del niño, de un rol o personaje ficcional distinto al propio. En la tabla 2 se detallan las situaciones de juego registradas en los hogares.

Como se observa en la tabla 2 , a los 2.6 predominan los juegos de comer, tomar el té y hablar por teléfono; en tanto que a los 3.6 se incrementan los porcentajes correspondientes a los juegos con muñecos o a otros juegos simbólicos que involucran un cambio de rol (Wilcoxon $₹=-5.06 p<.001 \mathrm{~N}=60$ ).

Ahora bien, ¿qué tipos de mundo ficcional construyen los niños y sus madres en las situaciones de juego identificadas? ¿Existen variaciones longitudinales en el tipo de mundo ficcional creado en el período comprendido entre los 2.6 y los 3.6 años? Con el fin de responder a estas preguntas se retomaron las categorías de Engel (2005) de what is -mundo ficcional que simula situaciones de la vida cotidiana- y what if -mundo ficcional que comprende elementos fantásticos-.

Como se observa en la tabla 3, los resultados del análisis longitudinal mostraron variaciones en el tipo de mundo ficcional creado en las situaciones lúdicas. En efecto, a los dos años y medio, los niños y sus madres creaban mayoritariamente mundos basados en eventos de la vida cotidiana, tales como llamar por teléfono a un familiar o tomar el té sin asumir otro rol; en tanto que, a los tres años y medio, se incrementa significativamente la proporción de situaciones en donde el mundo ficcional comprende eventos fantásticos en los que, por ejemplo, un brujo rapta a una princesa y un príncipe debe rescatarla (Wilcoxon $\%$ $=-4.71 p<.001 \mathrm{~N}=60$ ).

Con el objetivo de profundizar en el conocimiento de las situaciones de juego simbólico, se atendió a los recursos mediante los cuales los participantes construyen el mundo ficcional y la trama del juego. $\mathrm{El}$ análisis cualitativo puso de manifiesto que en los niños predomina la acción dramática acompañada con frecuencia de verbalizaciones que explicitan o especifican aspectos de la acción representada y del establecimiento de diálogos acordes con la situación representada.

\section{(1) Lautaro y su mamá (2.6) juegan a tomar el té.}

Madre: ¿Me preparás un té? Yo quiero un té con leche.

Niño: ¿Leche con miel?

Madre: Dale, leche con miel. Calentita por favor.

Lautaro simula preparar el té manipulando las tazas de juguete.

Niño: Estoy preparando.

Madre: ¿Qué rico!

Niño: Sí, rico.

Como se observa en el ejemplo, la madre inicia el juego proponiéndole al niño que le prepare un té. Por su parte, Lautaro participa en la situación lúdica llevando a cabo la acción dramática propuesta por su madre -Lautaro simula preparar el té manipulando las tazas de juguete- y explicita dicha acción con una verbalización -Estoy preparando- Tanto el niño como su madre establecen un diálogo ficcional que resulta acorde a la situación representada -Niño: ¿Leche con miel?, Madre: iQué rico!-. 
Tabla 2

Situaciones de juego registradas en los hogares

\begin{tabular}{lccc}
\hline & \multicolumn{2}{c}{ Edad de los niños } & Wilcoxon \\
\cline { 2 - 4 } Situaciones de juego & 2.6 años & 3.6 años & \\
\hline Jugar a comer / tomar el té & $16(47.1 \%)$ & $5(26.9 \%)$ & \\
Jugar a hablar por teléfono & $7(20.6 \%)$ & $9(34.7 \%)$ & \\
Juegos con muñecos & $5(17.6 \%)$ & $5(19.2 \%)$ & \\
Otros juegos dramáticos & $5(14.7 \%)$ & & \\
(implican un cambio de rol) & & $26(100 \%)$ & \\
\end{tabular}

Tabla 3

Tipo de mundo ficcional creado en la situación lúdica

\begin{tabular}{|c|c|c|c|c|}
\hline & 2.6 años & 3.6 años & Wilcoxon (z) & $p$ \\
\hline $\begin{array}{l}\text { Mundo que simula la vida } \\
\text { cotidiana (what is) }\end{array}$ & $29(85 \%)$ & $15(58 \%)$ & & .001 \\
\hline Mundo fantástico (what if) & $5(15 \%)$ & $11(42 \%)$ & -4.71 & \\
\hline Total & $34(100 \%)$ & $26(100 \%)$ & & \\
\hline
\end{tabular}

En algunos casos, se observó que el establecimiento del diálogo ficcional conlleva también el empleo del recurso lingüístico del cambio de registro (Halliday, 1979), que distingue las voces de los personajes de las voces de los participantes del juego, tal como se observa en el siguiente intercambio entre Paloma y su madre, quienes recurren al uso del usted, pronombre que no es empleado habitualmente en las comunicaciones entre madres e hijos de la población estudiada.

(2) Paloma (2.6) toma el juego de té.

Niña: ¿Qué quiere señora?

Mamá: No sé.

Niña: ¿Qué quiere usted, comida?

Madre: Bueno.
En cuanto a la participación de las madres, se observó que estas eran contingentes -respondían al foco de atención del niño-, intentaban sintonizarse con la actividad lúdica de los pequeños y aceptaban sus iniciativas. Los recursos empleados por las madres comprenden la denominación de los objetos que los niños manipulaban así como la verbalización de las acciones que conlleva la dramatización. En ocasiones, regulaban la construcción del mundo ficcional mediante directivas. Asimismo, explicitaban verbalmente el guión que orientaba la construcción de la situación representada y referían a situaciones habituales y a experiencias pasadas vinculadas con algún rasgo de la situación lúdica, como se observa en los fragmentos de intercambio que se presentan a continuación. 
(3) Mamá: ¿Vamos a tomar el té?

Niño: Sí, el té. Acá, ese, este es el té (toma la tetera y simula servirle té a su mamá).

(4) Nati, su mamá y su hermano juegan con un bebote de juguete.

Mamá: (Jugando con muñecos) Bueno, ¿a ver cómo le das la leche a Cata (bebote)?

Niña (2.6): Sin chupete.

Mamá: Claro, ¿quién usa chupete, Nati?

Niña: Bebés.

Mamá: ¿Los bebés? ¿Y vos usás?

Niña: No.

Mamá: ¿No? Mmm...

Hermano: Si que usa. Nati, sí que usás chupete.

Niña: No.

En fragmento (3) la madre explicita el guión que orienta la construcción del mundo ficcional del juego - ¿Vamos a tomar el té? la niña simula darle la mamadera a un muñeco, en tanto que su madre refiere a un hecho habitual relacionado con la situación lúdica: Nati usa chupete, igual que la muñeca.

En algunos casos, de manera incipiente, las madres y los niños distinguían entre el mundo ficcional y el no ficcional de manera explícita a través de enunciados metalúdicos en la planificación del juego.

(5) Candela y su madre juegan con muñecos.

Madre: ¿Y estos quiénes son? (señala los muñecos)

Niña: Son los papás.

Madre: Los papás.

Niña: Sí, esta es la camita.

Madre: ¿Qué? ¿Los papás duermen en el piso?

Niña: Dale que esta es la camita (señala un bloque).

En el ejemplo, la niña y su madre planifican determinados elementos del juego -los personajes, el escenario-, antes de comenzar a jugar. Ambas recurren a enunciados metalúdicos que, desde el mundo no ficcional, les permiten explicitar y negociar aspectos relativos al mundo ficcional del juego.
Dada la relevancia del uso de enunciados metalúdicos que se desprende de la revisión bibliográfica, se consideró la variación longitudinal de tales enunciados por parte de los participantes en las situaciones de juego. En la tabla 4, se observa un incremento significativo a los 3.6 años en la cantidad de situaciones de juego en las que los niños y sus madres emplearon enunciados metalúdicos en la construcción del mundo ficcional (Wilcoxon $z=6.69 p<.001$ ).

Finalmente, se analizó la estructura narrativa representada en la situación lúdica, con el fin de identificar las características de dicha trama, así como eventuales variaciones longitudinales durante el período considerado. Se identificó la presencia de los siguientes componentes de la trama narrativa del juego: identificación de personajes, caracterización de personajes, escenario, complicación, relación causal y de finalidad, relación temporal, solución o cierre.

Como se observa en la tabla 5 , en gran parte (38\%) de las situaciones registradas cuando los niños tenían 2.6 años, los participantes no refirieron de manera explícita a ningún componente estructural. En el $44 \%$ identificaron algún personaje, pero son poco frecuentes las situaciones en las que estos personajes desplegaban sus acciones en una trama que presentaba una complicación o relaciones temporales y causales entre los eventos representados. Solo en el 18\% de los casos, la madre y el niño atribuyeron alguna caracterización a los personajes y propusieron algún tipo de solución o cierre a la historia representada en la situación lúdica. Por el contrario, a los 3.6 años es mayor la proporción de situaciones en las que durante el juego los participantes identificaron de manera explícita algún componente estructural.

En el $73 \%$ de las situaciones, los niños y sus madres identificaron los personajes; en el 50\% de los casos refirieron al escenario en el que se desarrollaban las acciones y, en el 54\%, señalaron de manera explícita las relaciones causales entre los eventos representados. Como se observa en la tabla 5 los resultados de la prueba Wilcoxon muestran diferencias significativas según la edad en todos los componentes estructurales de la narrativa representada en el juego. 
Tabla 4

Situaciones de juego en las que se emplean enunciados metalúdicos en la construcción del mundo ficcional

\begin{tabular}{|c|c|c|c|c|}
\hline & 2.6 años & 3.6 años & Wilcoxon (z) & $p$ \\
\hline Ausencia de enunciados metalúdicos & $27(79 \%)$ & $14(54 \%)$ & 6.69 & .001 \\
\hline Uso de enunciados metalú dicos & $7(21 \%)$ & $12(46 \%)$ & & \\
\hline Total & $34(100 \%)$ & $26(100 \%)$ & & \\
\hline
\end{tabular}

Tabla 5

Componentes estructurales de las narrativas representadas en las situaciones lúdicas

\begin{tabular}{|c|c|c|c|c|c|}
\hline \multicolumn{2}{|c|}{ Componentes estructurales } & \multirow{2}{*}{$\frac{2.6 \text { años }}{13(38 \%)}$} & \multirow{2}{*}{$\begin{array}{c}3.6 \text { años } \\
2(8 \%)\end{array}$} & \multirow{2}{*}{$\frac{\text { Wilcoxon }(z)}{6.16}$} & \multirow{2}{*}{$\frac{p}{.001}$} \\
\hline No se explicitan componentes & & & & & \\
\hline Identificación de personajes & $\begin{array}{l}\text { Niño: Esta es la hija, este es el } \\
\text { papá y esta es la mamá. }\end{array}$ & $15(44 \%)$ & $19(73 \%)$ & 6.06 & .001 \\
\hline Caracterización de personajes & $\begin{array}{l}\text { Madre: Bueno, es un cocodrilo. } \\
\text { Mientras no nos coma a } \\
\text { nosotros... Niño: No. Es un } \\
\text { cocodrilo bueno. }\end{array}$ & $6(18 \%)$ & $10(38 \%)$ & 6.81 & .001 \\
\hline Escenario & $\begin{array}{l}\text { Madre: Vamos a hacer la casa. } \\
\text { Esta es la casa. La habitación, } \\
\text { la cocina y el garage. }\end{array}$ & $8(24 \%)$ & $13(50 \%)$ & 6.71 & .001 \\
\hline Complicación & $\begin{array}{l}\text { Niño: Dale que se casan y la } \\
\text { roba alguien a Olivia... La } \\
\text { quiere raptar el capitán del barco. }\end{array}$ & $10(29 \%)$ & $11(42 \%)$ & 6.51 & .001 \\
\hline Relación causal / de finalidad & $\begin{array}{l}\text { Niño: No hay que despertar al } \\
\text { lobo porque si no, se despierta y } \\
\text { los come. }\end{array}$ & $9(27 \%)$ & $14(54 \%)$ & 6.64 & .001 \\
\hline Relación temporal & $\begin{array}{l}\text { Madre: (habla el personaje) } \\
\text { Entonces primero me voy al } \\
\text { cumpleaños y después los veo más } \\
\text { tarde. }\end{array}$ & $8(24 \%)$ & $7(27 \%)$ & 6.63 & .001 \\
\hline Solución / Cierre & $\begin{array}{l}\text { Niño: Dale que se casan y la } \\
\text { roba alguien a Olivia y Ulises } \\
\text { la salva. }\end{array}$ & $6(18 \%)$ & $9(35 \%)$ & 6.8 & .001 \\
\hline Total & & 34 situaciones & 26 situaciones & & \\
\hline
\end{tabular}

Nota. Las categorías presentadas no son excluyentes entre sí. La tabla informa la presencia de cada componente narrativo explicitado verbalmente en las situaciones de juego analizadas. 


\section{Discusión}

El presente estudio se focalizó en el análisis del mundo ficcional creado en situaciones de juego simbólico, los recursos empleados por los niños y sus madres para construirlo y las características de la trama narrativa representada durante el período comprendido entre los 2.6 y 3.6 años. Específicamente, se buscó responder a los siguientes interrogantes: ¿Qué tipos de mundo ficcional involucran los juegos en los que participan los niños a los 2.6 y a los 3.6 años? ¿Cuáles son los recursos mediante los cuales los niños y sus interlocutores construyen el mundo ficcional y la trama del juego? ¿Qué características asume la trama narrativa del mundo ficcional del juego en esta etapa?

En relación con el tipo de mundo ficcional creado en el juego, los resultados mostraron que a los 3.6 años tiene lugar un incremento en las situaciones en las que los niños y sus compañeros de juego crean mundos ficcionales que comprenden elementos fantásticos (what if). Este hallazgo coincide con los estudios previos, que mostraron que los primeros juegos simulan situaciones familiares para los niños y que, progresivamente, incorporan una mayor diversidad de situaciones y personajes más alejados de la experiencia cotidiana infantil (Engel, 2005; Garvey, 1985; Garvey \& Brent, 1977; Schwartzman, 1978; Westby, 1988).

Para crear el mundo ficcional del juego, los resultados del análisis presentado evidenciaron que los niños y sus madres emplean recursos diversos. En el juego, los niños y sus madres recurren a la dramatización de acciones; a verbalizaciones que explicitan aspectos de la situación representada; al establecimiento de diálogos desde la perspectiva de los personajes; al empleo de directivas; a la explicitación del guión representado; al uso de enunciados metalúdicos y a la referencia a situaciones habituales y pasadas temáticamente similares a la acción dramática. Asimismo, se observó el empleo del recurso lingüístico del cambio de registro para diferenciar los planos del juego. Estos recursos son similares a los observados en otros trabajos (Andresen, 2005; Garvey, 1985; Garvey \& Kramer, 1989; Gönçü, 1987; Rubin et al., 1983; Sawyer, 2002).
En particular, en cuanto al uso de enunciados metalúdicos por parte de los niños en la gestión del mundo ficcional, varios trabajos han observado su presencia en situaciones de juego de las que participan niños mayores de 3 años (Andresen, 2005; Garvey \& Kramer, 1989; Gönçü, 1987; Migdalek, Rosemberg \& Arrúe, 2013, 2014; Rubin et al., 1983; Sawyer, 2002).

En cambio, se observó que algunos de los niños participantes en este estudio hacían un uso incipiente de tales enunciados a los 2.6 años. Es posible pensar que el uso temprano observado en el presente trabajo se deba al hecho de que los niños interactúan, en las situaciones de juego analizadas, con un adulto que puede modelar el empleo de este recurso (Nielsen \& Christie, 2008; Rakoczy, 2007; 2008). El incremento significativo del empleo de enunciados metalúdicos en las situaciones registradas cuando los niños tienen 3.6 años coincide con los resultados de Garvey y Kramer (1989) y de Andresen (2005).

Tal como ha sido señalado en los resultados, los niños recurren a la dramatización de acciones y el establecimiento de diálogos ficcionales a partir de la voz de los personajes. Sawyer (2002) observó que, cuando en el marco de estos diálogos tiene lugar una negociación acerca del curso del juego, ello contribuye a la elaboración de la trama narrativa. Por el contrario, en los casos en que dicha negociación no tiene lugar, ello repercute negativamente en la complejidad de la trama narrativa del juego. En el presente estudio, a diferencia del trabajo de Sawyer (2002), como se trata de interacción adulto-niño siempre hay una negociación en tanto el adulto trata de sintonizar con la intención lúdica del niño.

En cuanto a las características de la trama narrativa del juego durante el período considerado, los resultados mostraron un incremento significativo de la complejidad narrativa a los 3.6 años. En efecto, a esa edad, se observó una mayor explicitación verbal de todos los componentes narrativos de la trama lúdica y de las relaciones entre ellos mediante el establecimiento de relaciones causales, temporales y de finalidad. Este incremento en la complejidad narrativa del juego coincide con las investigaciones antecedentes; 
mostraron que los niños mayores no representan acciones aisladas sino que, progresivamente, pueden entrelazarlas en una secuencia narrativa (Elkonin, 1980; Westby, 1988).

En conjunto, los resultados del análisis longitudinal mostraron, por un lado, diferencias respecto del rol que cumple el lenguaje en la estructuración narrativa del juego. Tal como se señaló anteriormente, se observó un incremento en el empleo de enunciados metalúdicos; esto es, afirmaciones explícitas acerca del juego, en las situaciones registradas a los 3.6 años. Simultáneamente, a esta edad se registró una mayor complejidad narrativa reflejada en la explicitación verbal de cada uno de los componentes narrativos.

Por otro lado, una mayor proporción de las situaciones registradas a los 3.6 involucran mundos ficcionales fantásticos. Cabe suponer que el incremento de estos tres aspectos -empleo de enunciados metalúdicos, variedad de componentes narrativos en la trama del juego y el despliegue de un mundo ficcional con elementos fantásticos- se interrelacionan y potencian entre sí. En particular, el uso de enunciados metalúdicos ha sido señalado como un elemento destacado para la constitución del mundo ficcional (Sawyer, 2002; Rosemberg, Arrúe \& Migdalek, 2015). Su aumento con la edad, de manera simultánea con un descenso de la mera acción dramática, posibilita la creación de mundos ficcionales más variados, alejados de la vida cotidiana, así como la elaboración de tramas narrativas estructuralmente más complejas.

En conjunto, los resultados del presente trabajo proporcionan nueva evidencia que reafirma la relación bidireccional entre juego y lenguaje (Rosemberg, 2008) en general y entre juego y habilidades narrativas en particular (Galda, 1984; Nicolopoulou et al., 2015; Pellegrini, 1985a). Asimismo, aportan información relevante acerca de las características que asume la construcción del mundo ficcional del juego en poblaciones de habla hispana, a edades tempranas. Adicionalmente, informan sobre las variaciones que dichas características asumen a lo largo del desarrollo. En investigaciones futuras, se continuará profundizando en el estudio de las variaciones en los recursos según la edad de los niños y en el tipo de mundo ficcional representado (cercano versus alejado de la vida cotidiana), atendiendo discriminadamente a la participación infantil y del adulto en las situaciones de juego. En el análisis, se contemplarán también momentos posteriores en el desarrollo, hasta los 5 años de edad.

\section{Referencias}

Andresen, H. (2005). Role play and language development in the preschool years. Culture Psychology, 11(4), 387-414. doi: 10.1177/1354067X05058577

Auza, A. (2013). La mirada sociocultural en las prácticas narrativas de niños hispanohablantes. Actualidades en Psicología, 27(115), 141-146. doi: 10.15517/ap.v27i115.12182

Bruner, J. (1986). El habla del niño. Barcelona: Paidós.

Elkonin, D. B. (1980). Psicología del juego. Madrid: Visor Aprendizaje.

Engel, S. (2005). The narrative worlds of what is and what if. Cognitive Development, 20(4), 514-525. doi: 10.1016/j.cogdev.2005.08.005

Fein, G. G., Ardila-Rein, A. E., \& Groth, L. A. (2000). The narrative connection: Stories and literacy. In K. A. Roskos \& J. F. Christies (Eds.), Play and literacy in early childhood (pp. 27-43). Mahwah, NJ: Erlbaum.

Feldman, C. F. (2005). Mimesis: Where play and narrative meet. Cognitive Development, 20(4), 503-513. doi: 10.1016/j.cogdev.2005.08.006

Galda, L. (1984). Narrative competence: Play, storytelling and story comprehension. In A. Pellegrini \& T. Yaukey (Eds.), The development of oral and written language in social contexts (pp. 105-117). Norwood, NJ: Ablex.

Garvey, C. (1985). El juego infantil. Madrid: Morata.

Garvey. C. \& Berndt, R. (1977). The organization of pretend play. JSAS Catalog of Selected Documents in Psychology, 7 (Ms. No. 1589).

Garvey, C., \& Kramer, T. L. (1989). The language of social pretend play. Developmental Reviem, 9(4), 364382. doi: 10.106/0273-2297(89)90035-X 
Glaser B. \& Strauss, A. (1967). The discovery of grounded theory: strategies for qualitative research. New York: Aldine Publishing Company.

Gönçü, A. (1987). Toward an interactional model of developmental changes in social pretend play. In L. Katz (Ed.), Current topics in early childhood education (Vol. 7, pp. 108-125). Norwood, NJ: Ablex.

Gönçü, A. \& Gaskins, S. (2011). Comparing and extending Piaget's and Vygotsky's understandings of play: Symbolic play as individual, sociocultural, and educational interpretation. In Pellegrini, A.D. (Ed.), Oxford handbook of the development of play (pp. 48-57). Oxford: Oxford University Press.

Halliday, M. A. K. (1979). El lenguaje como semiótica social. México: FCE.

Ilgaz, H., \& Aksu-Koc, A. (2005). Episodic development in preschool children's play-prompted and direct-elicited narratives. Cognitive Development, 20(4), 526-544. doi: 10.1016/j.cogdev.2005.08.004

Kavanaugh, R., \& Engel, S. (1998). The development of pretense and narrative in early childhood. En O. Saracho \& B. Spodek (Eds.), Multiple perspectives on play in early childhood education (pp. 80-99). Albany: State University of New York Press.

Leslie, A. (1987). Pretense and representation: The origins of "theory of mind". Psychological Review, 94(4), 412-426. doi: 10.1037/0033-295X.94.4.412

Migdalek, M., Rosemberg, C. R., \& Arrúe, J. (2013). La elaboración lingüística del marco ficcional en el juego dramático. Variaciones entre niños de distintos grupos sociales. RASAL, 101-117.

Migdalek, M., Santibáñez, C., \& Rosemberg, C. R. (2014). Estrategias argumentativas en niños pequeños: Un estudio a partir de las disputas durante el juego en contextos escolares. Revista Signos. Estudios de Lingüistica, 47(86), 435-462. doi: 10.4067/S0718-09342014000300005

Nelson, K. (1996). Language in cognitive development: The emergence of mediated mind. New York: Cambridge University Press.
Nicolopoulou, A., McDowell, J., \& Brockmeyer, C. (2006). Narrative play and emergent literacy: Storytelling and story-acting meet journal writing. En D. G. Singer, R. Michnik \& K. Hirsh-Pasek (Eds.), Play = Learning (pp. 124-144). New York: Oxford University Press.

Nicolopoulou, A., Schnabel Cortina, K., Ilgaz, H. \& Brockmeyer, A. B. de Sá. (2015). Using a narrativeand play-based activity to promote low-income preschoolers' oral emergent literacy and social competence. Early Childhood Research Quarterly, 31(2), 147-162. doi: 10.1016\&/j.ecresq.2015.01.006

Nielsen, M., \& Christie, T. (2008). Adult modelling facilitates young children's generation of novel pretend acts. Infant and Child Development, 17(2), 151162. doi: $10.1002 /$ icd. 538

Ortega, R. (1994). El juego sociodramático como contexto para la comprensión social. En P. Del Río, A. Álvarez \& J. Wertsch (Eds.), Explorations in Socio-Cultural Studies: Education as cultural construction (pp. 79-86). Madrid: Fundación Infancia y Aprendizaje.

Paley, V. (1990). The boy who would be a belicopter: The uses of storytelling in the classroom. Cambridge, MA: Cambridge University Press.

Pellegrini, A. (1982). The construction of cohesive text by preschoolers in two play contexts. Discourse Processes, 5(1), 101-108. doi: 10.1080/01638538209544533

Pellegrini, A. (1984). Effects of experimental play contexts on the development of preschoolers' functional language. Journal of pragmatics, 8(2), 211219. doi: 10.1016/0378-2166(84)90050-X

Pellegrini, A. D. (1985a). The narrative organization of children's fantasy play. Educational Psychology, 5(1), 17-25. doi: 10.1080/0144341850050103

Pellegrini, A. D. (1985b). The relations between symbolic play and literate behavior: a review and critique of the empirical literature. Review of Educational Research, 55(1), 107-121. doi: 10.3102/00346543055001107 
Pellegrini, A. (1986). Play Centers and the Production of Imaginative Language. Discourse Processes, 9(1), 115-125. doi: 10.1080/016385386095544634

Pellegrini, A. D., \& Galda, L. (1982). The effects of thematic fantasy play training on the development of children's comprehension. American Educational Research Journal, 19(3), 443-452. doi: 10.3102/00028312019003443

Petersen, D. B., Gillam, S. L., \& Gillam, R. B. (2008). Emerging procedures in narrative assessment. The index of narrative complexity. Topics in language disorders, 28(2), 115-130. doi: 10.1097/01. TLD.0000318933.46925.86

Peterson C., \& McCabe, A. (1983). Developmental psycholinguistics: Three ways of looking at a child's narrative. New York: Plenum.

Rakoczy, H. (2007). Play, games, and the development of collective intentionality. New Directions for Child and Adolescent Development, 115, 53-67. doi: 10.1002/cd.182

Rakoczy, H. (2008) Pretense as Individual and Collective Intentionality. Mind \& Language, 23(5), 499-517. Doi: 10.1111/j.1468-0017.2008.00357.x

Rosemberg, C. R. (2008). El lenguaje y el juego en la educación infantil. En P. Sarlé (comp.) Enseñar en clave de juego enlazando juegos y contenidos de enseñanza (pp. 61-75). Buenos Aires: Novedades Educativas.

Rosemberg, C. R., Arrúe, J., \& Migdalek, M. (2015). El lenguaje en la construcción del "mundo de ficción” en el juego dramática. En P. M. Sarlé \& C. R. Rosemberg (coords.), Dale que... El juego dramático y el desarrollo del lenguaje en los niños pequeños (pp. 109-123). Buenos Aires: Homo Sapiens.

Rubin, K., Fein, G., \& Vandenberg, B. (1983). Play. In E. Hetherington (Ed.), P. H. Mussen (Series Ed). Handbook of child psychology: Vol. 4. Socialization, personality and social development (pp. 693-774). New York: Wiley.
Sachs, J., Goldman, J., \& Chaille, C. (1984). Planning in pretend play: Using language to coordinate narrative development. En A. Pellegrini \& T. Yawkey (Eds.), The development of oral and written language in social contexts (pp. 119-128). Norwood, NJ: Ablex.

Sarlé, P., \& Rosas, R. (2005). Juegos de construcción y construccióndelconocimiento. Buenos Aires:MiñoyDávila.

Sawyer, R. K. (2002). Improvisation and narrative. Narrative Inquiry, 12(2), 319-349. doi: 10.1075/ ni.12.2.05saw

Schwartzman, H. (1978). Transformations: The anthropology of children's play. New York: Plenum.

Stein, A., Migdalek, M., \& Sarlé, P. (2012). "Te enseño a jugar": Caracterización de movimientos interaccionales y formas lingüísticas mediante las cuales se regula la interacción lúdica. Psykhe, 21(1), 55-67. doi: 10.4067/S0718-22282012000100004

Stein, N. L., \& Glenn, C. G. (1978). An analysis of story comprehension in elementary school children. En R. O. Freedle (Ed.), New directions in discourse processing (pp. 53-120). Norwood, NJ: Ablex.

Stein, N. L., \& Glenn, C. G. (1982). Children's concept of time: The development of a story schema. En W. Friedman (Ed.), The developmental psychology of time (pp. 255-282). New York: Academic Press.

Strauss, A., \& Corbin, J. (1991). Basics of qualitative research. Londres: Sage.

Trionfi, G., \& Reese, E. (2009). A good story: Children with imaginary companions create richer narratives. Child Development, 80(4), 1301-1313. doi: 10.1111/j.1467-8624.2009.01333.x

Vigotsky, L. S. (1978). El desarrollo de los procesos psicológicos superiores. Barcelona: Grijalbo.

Westby, C. E. (1988). Children's play: Reflections of social competence. Seminars in Speech and Language, 9(1), 1-14. doi: 10.1055/s-2008-1064445 
